\title{
Numerical prediction of the focal sites of ozone-induced tissue injury in the respiratory tract
}

\author{
B. Keshavarzi, J. Ultman \& A. Borhan \\ Department of Chemical Engineering, The Pennsylvania State University, \\ USA
}

\begin{abstract}
Numerical simulations of ozone transport and uptake in an anatomically-accurate model of the respiratory tract of a Rhesus monkey were performed. The model geometry was created using three-dimensional reconstruction of MRI images of the respiratory tract, including the larynx and the first thirteen generations of the tracheobronchial tree. An unstructured mesh was generated for the resulting structure, and three-dimensional flow and concentration distributions were obtained through numerical solution of the Navier-Stokes, continuity, and species convection-diffusion equations. A quasi-steady diffusion-reaction model was used to account for the interaction between $\mathrm{O}_{3}$ and endogenous substrates in the respiratory tract lining fluid. Hotspots of $\mathrm{O}_{3}$ flux on the walls were identified for steady inspiratory flow under quiet breathing conditions.
\end{abstract}

Keywords: ozone, uptake, respiratory tract, larynx, conducting airways.

\section{Introduction}

Ozone $\left(\mathrm{O}_{3}\right)$ is a highly reactive gas and a harmful air pollutant. Ground level ozone is formed primarily by the action of sunlight on hydrocarbon vapors and nitrogen oxides emitted by combustion of fossil fuels. It can irritate the respiratory tract, reduce lung function, and increase the frequency of asthma attacks in populations suffering from asthma. Recent observations have shown that exposure to ozone can also produce intense remodeling in the developing lungs of infant primates, resulting in the loss of conducting airways [1]. The national ambient air quality standard for $\left(\mathrm{O}_{3}\right)$ level is $0.075 \mathrm{ppm}$ averaged over eight hours. However, tests 
carried out on a group of healthy adults and children undergoing moderate exercise have shown that even lower levels of ozone can cause a decrease in breathing ability [2].

The toxicity of oxidant gases such as ozone is believed to be due to peroxidation of membrane lipids, leading to destruction of membrane integrity and eventual cell death. Ozone may also oxidize reduced sulfhydryl groups of proteins and peptides such as Glutathione [3]. Absorption of ozone occurs in all regions of the respiratory tract, and the pattern of lung injury induced by the inhalation of ozone appears to depend on the dose delivered to different tissues in the airways. For example, Castleman et al. [1] performed controlled $\mathrm{O}_{3}$ exposures of bonnet monkeys, and observed epithelial cell hyperplasia and macrophage accumulation that were primarily focused in the proximal alveolar region. They also reported less severe epithelial damage in the trachea and large bronchi, and little or no damage in other lung regions. Similarly, Chang et al. [4] found that the degree of focal injury occurring in the proximal alveolar region of Fisher-344 rats was directly dependent on the integrated $\mathrm{O}_{3}$ concentration-time profile of the inhaled gas. Thus, regional differences in cell injury are probably related to corresponding differences in the rates of $\mathrm{O}_{3}$ delivery.

Although local dose cannot be easily measured, especially in a site-specific manner, mathematical dosimetry models can provide predictions of the uptake and distribution of absorbed gases such as $\mathrm{O}_{3}$ in the respiratory tract [5,6]. Dosimetry refers to the estimation or measurement of the amount of a compound (or its toxic metabolites or reaction products) that reaches specific target sites after exposure to a given concentration of the compound. While it is critical to understand interspecies differences in the respiratory tract in order to assess the implications of toxicological results from animal studies to humans, dosimetry modeling can also play an extremely important role. Previous analysis of transport and removal of $\mathrm{O}_{3}$ in the lungs of guinea pigs, rabbits, and humans indicates the existence of a general similarity among these species in the shapes of the dose curves [7]. Thus, accurate dosimetry models that incorporate physical, biological and chemical properties of the respiratory tract, as well as the nature of gas transport in the lumen and air spaces, can serve as invaluable predictive tools in the extrapolation of animal toxicological results to humans [8].

The development of $\mathrm{O}_{3}$ dosimetry models for laboratory animals and humans has mainly focused on the effects of $\mathrm{O}_{3}$ in the lower respiratory tract $(L R T)$ comprising the tracheobronchial tree which includes the trachea and a series of branching tubes that become narrower, shorter, and more numerous before ending at the terminal bronchioles (cf. Recent advances in the development of reliable algorithms for three-dimensional reconstruction of complex geometries, combined with well-established computational fluid dynamics (CFD) strategies for the computation of flows in such geometries, have paved the way for the development of more sophisticated dosimetry models based on anatomicallyaccurate geometries of the respiratory tract. In this paper, we present the results of three-dimensional simulations of ozone transport and uptake during inspiratory 
flow in an anatomically-accurate model of the $L R T$ of a rhesus monkey that also includes the larynx.

\section{Methods}

\subsection{Airway geometry}

Based on the structural similarity between the human and rhesus monkey respiratory tracts, the respiratory tract of a rhesus monkey was selected as the platform for this computational study. In addition, experimental assessment of ozoneinduced injury in the conducting airways of rhesus monkeys is currently being conducted, thereby allowing direct comparison between theoretical predictions and experimental observations in the near future.

The anatomically-accurate airway geometry was created from 3-D reconstructions of the larynx and tracheobronchial tree using MRI data for the casts of the respective parts of a rhesus monkey. The MRI data consisted of 256 transverse slices depicting square fields of view $10 \mathrm{~cm}$ on a side for the tracheobronchial tree and $3.5 \mathrm{~cm}$ on a side for the larynx. The thickness of each slice and the resolution of its sides were both 391 microns for the tracheobronchial tree, and 137 microns for the larynx. All slices were stored as 8-bit TIFF (Tag Image File Format) files containing $256 \times 256$ pixels in $D D V$ (Digital Data Viewer) format.

The MRI images were imported as raw data into the three-dimensional visualization and volume modeling software, AMIRA (Mercury Computer Systems, Chelmsford, MA). Open-ended triangulated surfaces for the larynx and the tracheobronchial tree were created in AMIRA and exported to the commercial meshing software GAMBIT (ANSYS, Canonsburgh, PA). The extraneous lowresolution parts of small branches were cut off from the geometry, and the geometry was cleaned up by removing hard and short edges, as well as small faces. Closed volumes for the larynx and the tracheobronchial tree were then created from their respective cleaned-up surfaces, and the two volumes were subsequently attached together. The resulting structure included thirteen airway generations beyond the larynx. Accurate reconstruction beyond the thirteenth generation would require a better resolution of the $M R I$ images.

The entire airway geometry was meshed using tetrahedral elements of dimensionless size 0.05 (made dimensionless with the hydraulic radius of the trachea). Zone types such as inflow, outflow and wall were assigned to the bounding surfaces, and the meshed geometry was exported to the commercial CFD software FLUENT 6.3 (ANSYS, Canonsburgh, PA) for the simulations.

\subsection{Mathematical formulation}

Simulations were performed for steady incompressible laminar flow of a binary gas-mixture of air and ozone. The flow field is governed by dimensionless continuity and Navier-Stokes equations given by

$$
\nabla \cdot \mathbf{u}=0
$$




$$
\frac{\partial \mathbf{u}}{\partial t}+\mathbf{u} \cdot \nabla \mathbf{u}=-\nabla p+\frac{1}{R e} \nabla^{2} \mathbf{u}
$$

where $\mathbf{u}$ and $p$ are the dimensionless velocity and dynamic pressure, respectively, and $\nabla$ is the dimensionless gradient operator. All lengths are made dimensionless with the hydraulic radius of the trachea, $R_{h}$, velocities with $U_{0}=Q_{0} / \pi R_{h}^{2}$, time with $R_{h} / U_{0}$, and pressure with $\rho U_{0}^{2}$, where $\rho$ denotes the density of the gas mixture, and $Q_{0}$ denotes the volumetric flow rate through the trachea. The Reynolds number, $R e=U_{0} R_{h} / v$, appearing in Eq. 2 represents the tracheal Reynolds number, with $v$ denoting the kinematic viscosity of the gas mixture.

Equations (1) and (2) are solved subject to the following boundary conditions:

- Uniform velocity normal to the inflow plane of the larynx for inspiratory flow.

- Zero velocity $(\mathbf{u}=0)$ at the airway walls (no-slip condition).

- Zero viscous normal stress and prescribed volumetric flow rates at the outflow boundaries, with the flow splits among outflow boundaries specified to be proportional to their cross-sectional areas.

The $\mathrm{O}_{3}$ concentration distribution in the gas mixture is governed by the dimensionless convection-diffusion equation, which can be written as

$$
\frac{\partial C}{\partial t}+\mathbf{u} \cdot \nabla C=\frac{1}{P e} \nabla^{2} C,
$$

where the concentration $C$ is made dimensionless with the inlet $\mathrm{O}_{3}$ concentration, $C_{0}, P e=U_{0} R_{h} / D_{g}$ is the tracheal Peclet number, and $D_{g}$ denotes the gas phase diffusivity of $\mathrm{O}_{3}$. The boundary conditions for this equation include:

- Uniform $\mathrm{O}_{3}$ concentration, $\mathrm{C}=1$, at the inflow boundary.

- Zero diffusive flux, $\mathbf{n} \cdot \nabla C=0$, at the outflow boundaries

- An appropriate diffusion-reaction condition at the airway wall, based on the specific model used for the reaction of $\mathrm{O}_{3}$ within the respiratory tract lining fluid $(R T L F)$

The last boundary condition considers either an infinitely fast or a slow firstorder reaction of $\mathrm{O}_{3}$ with substrates in the $R T L F$. Hence, several different reactions can take place between $\mathrm{O}_{3}$ and the $R T L F$ components. In the absence of reliable data for the rates of these reactions, we have to rely on simplified models for the reactions between $\mathrm{O}_{3}$ and $R T L F$ substrates. The simplest model that is considered in our simulations is that of an infinitely fast reaction. In this model, the rate of reaction is so fast (compared to the rate of $\mathrm{O}_{3}$ transport to the $R T L F$ ) that the $\mathrm{O}_{3}$ concentration vanishes at the gas- $R T L F$ interface, leading to the boundary condition $C=0$ at the airway wall. Although using an infinite reaction rate overestimates the rate of $\mathrm{O}_{3}$ uptake, it also magnifies the non-uniformity in the wall flux distribution, and facilitates the identification of hot spots of $\mathrm{O}_{3}$ flux (which is one of the main objectives of this study).

In reality, the rate of reaction of $\mathrm{O}_{3}$ in the $R T L F$ is finite. Hence, a second model for the reaction between $\mathrm{O}_{3}$ and $R T L F$ substrates can be formulated based on the assumption that the substrate concentrations in the RTLF are much higher than $\mathrm{O}_{3}$ concentrations that can result from any reasonable $\mathrm{O}_{3}$ exposure. In that 
case, the reaction can be viewed as a pseudo-first order reaction with respect to $\mathrm{O}_{3}$ concentration in the $\mathrm{RTLF}$. Neglecting convection in the $R T L F$, the quasisteady diffusion and reaction of $\mathrm{O}_{3}$ within the $R T L F$ is described by the species conservation equation

$$
\nabla^{2} c=D a^{2} c
$$

where $c$ is the $O_{3}$ concentration in the $R T L F$ (made dimensionless with $C_{0}$ ). The Damkohler number, $D a=\sqrt{\frac{k_{r} \Delta^{2}}{D_{l}}}$, represents the ratio of the characteristic time for diffusion to that for chemical reaction, with $k_{r}$ denoting the rate constant for the pseudo-first order reaction, $\Delta$ the thickness of the $R T L F$ layer, and $D_{l}$ the diffusivity of $\mathrm{O}_{3}$ in the $R T L F$.

The thickness of the RTLF layer is typically much smaller than the airway radius. It decreases with longitudinal position from about $10 \mu \mathrm{m}$ in the upper airways to about $0.1 \mu \mathrm{m}$ in the respiratory zone, while the airway radii vary in the range 0.02-0.09 $\mathrm{cm}$ depending on the airway generation [9]. As such, a planar (rather than annular) view of the RTLF can be adopted in conjunction with the lubrication approximation to reduce Eq. (4) to

$$
\frac{\partial^{2} c(y, z)}{\partial y^{2}}=D a^{2} c(y, z)
$$

where $y$ and $z$ denote the coordinates (made dimensionless with $\Delta$ ) normal and tangent to the gas- $R T L F$ interface, respectively.

The boundary conditions consist of vanishing $\mathrm{O}_{3}$ concentration at the $R T L F$ tissue interface, and local equilibrium between the $\mathrm{O}_{3}$ concentrations on the two sides of the gas-RTLF interface. The latter can be expressed as $c=\alpha C$, where $\alpha$ is the equilibrium partition coefficient. The solution of Equation (4) subject to these boundary conditions yields the following $\mathrm{O}_{3}$ concentration profile within the RTLF:

$$
c(y, z)=\frac{\alpha C(z) \sinh [D a(1-y)]}{\sinh (D a)}
$$

The resulting expression for the dimensionless $\mathrm{O}_{3}$ flux into the $R T L F$ layer is given by

$$
N=-\left.\frac{\partial c}{\partial y}\right|_{y=0}=-\alpha C(z) D a \operatorname{coth}(D a) .
$$

Requiring that the $\mathrm{O}_{3}$ flux be continuous across the gas- $R T L F$ interface then leads to the effective wall reaction condition

$$
N=-\mathbf{n} \cdot \nabla C=K C ; K=\left(\frac{D_{l}}{D_{g}}\right)\left(\frac{R}{\Delta}\right) \alpha D a \operatorname{coth}(D a),
$$

which can be used as a boundary condition on the airway walls for Eq. (3) in simulations based on the pseudo-first order reaction model. 


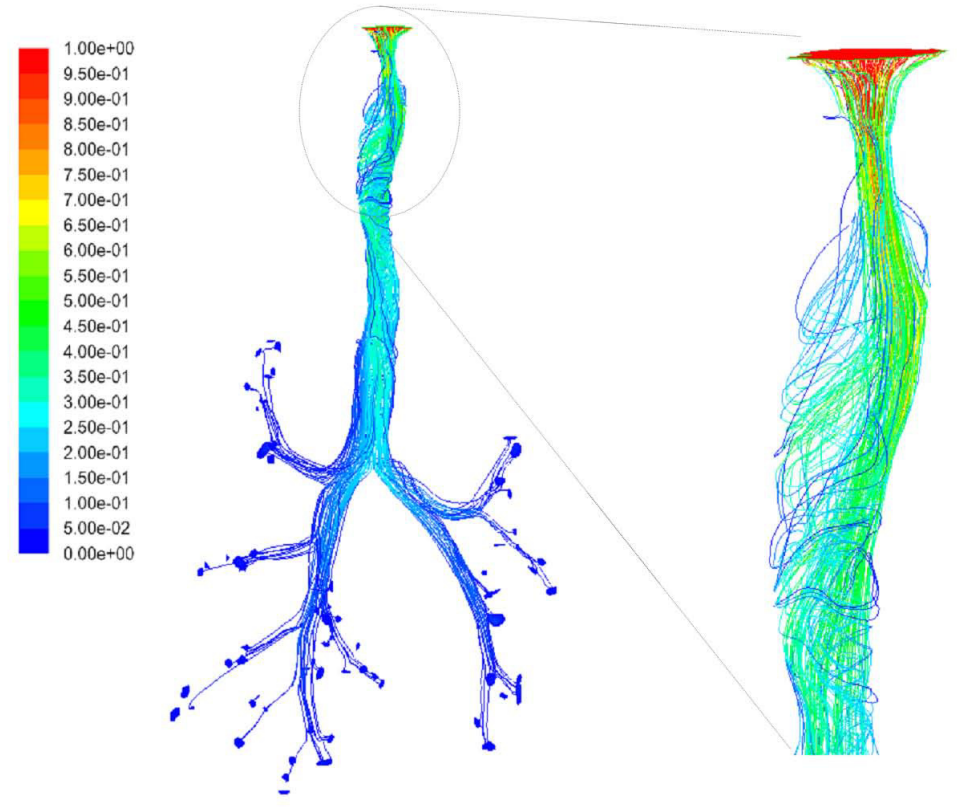

Figure 1: Streaklines of flow, color-labeled by local $\mathrm{O}_{3}$ concentration, for an infinitely-fast reaction $(R e=230, P e=195)$.

\section{Results}

Using the reported results for the daily air intake of a monkey based on its body weight [10], a range of inspiratory flow rates was estimated for rhesus monkeys. Simulations were performed for a tracheal Reynolds number of 230, corresponding to the upper end of the range of Reynolds numbers for quiet breathing condition. Based on a Schmidt number of $S c=0.85$ for the ozone/air mixture [11], the corresponding value of Peclet number in the simulations was 195 . For the pseudofirst order reaction model, a value of $D a=15$ was used is the simulations, corresponding to a rate constant on the order of $10,000 \mathrm{~s}^{-1}$ which represents the lower end of the values reported in the literature. Proper convergence and accuracy of the computations were verified by performing the requisite mesh refinement studies.

Streaklines of flow, color-labeled by the local $\mathrm{O}_{3}$ concentration (with red representing high concentration and blue denoting low concentration), are shown in Figure 3 for an infinitely-fast reaction. In this case, the $\mathrm{O}_{3}$ concentration at most of the outflow boundaries is nearly zero, and very little ozone leaves through 


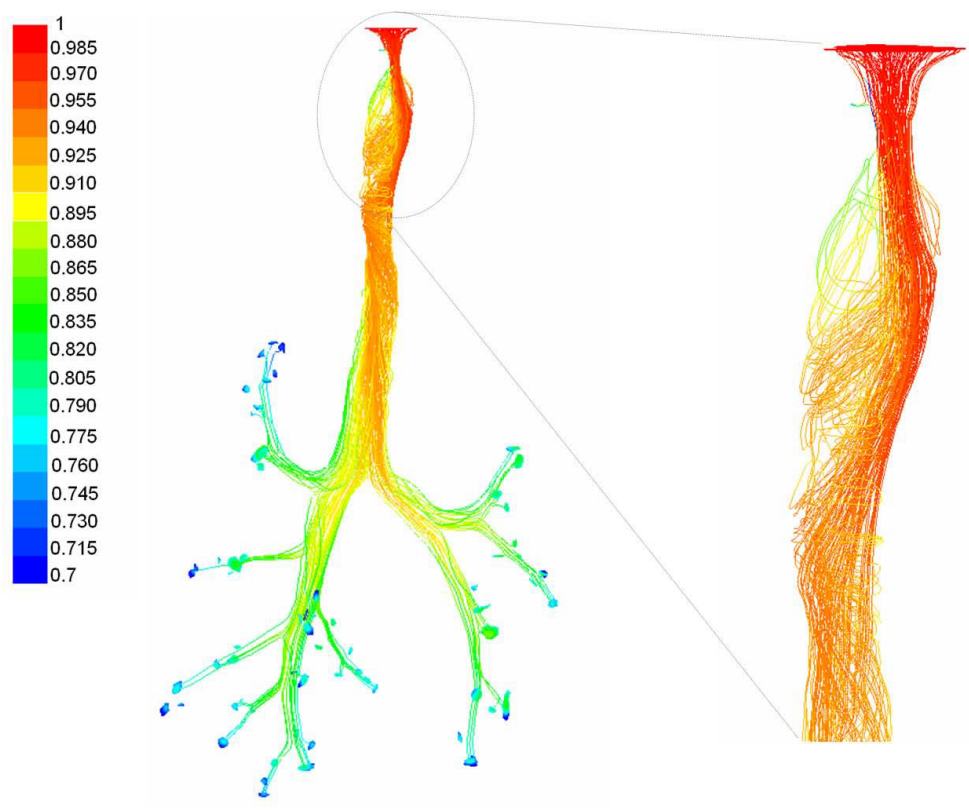

Figure 2: Streaklines of flow, color-labeled by local $\mathrm{O}_{3}$ concentration, for a slow first-order reaction with $D a=15(R e=230, P e=195)$.

the distal airways. The corresponding results for a slow first-order reaction with $D a=15$, but otherwise identical values of the dimensionless parameters, are shown in Figure 3. In contrast to the case of an infinitely fast reaction, a significant amount of ozone remains unreacted in the airways even after thirteen generations along the main path. In this case, most of the $\mathrm{O}_{3}$ is transported to the airways beyond the distal end of the airway structure considered here.

In the absence of the larynx, local maxima in $O_{3}$ wall flux occur just downstream of the carina of each bifurcation (specially in the first three bifurcations of the conducting airways), with the magnitudes of the spikes decreasing with each branching generation $[5,11]$. The appearance of the spikes in wall flux just downstream of bifurcations is due to the development of thin concentration boundary layers on the airway walls, starting from the carina. As the flow enters downstream branches, the local Reynolds number of the flow decreases. At lower Reynolds number, the thickness of the concentration boundary layer on the airway wall is larger at any given distance downstream of the carina, thereby leading to a smaller $\mathrm{O}_{3}$ flux at the airway wall. As expected, the magnitudes of the hotspots of $\mathrm{O}_{3}$ flux for the infinitely-fast reaction are much larger than those for the slow firstorder reaction. While the hotspots of $\mathrm{O}_{3}$ wall flux just downstream of the airway bifurcations persist even in the presence of the larynx, their intensities are reduced 

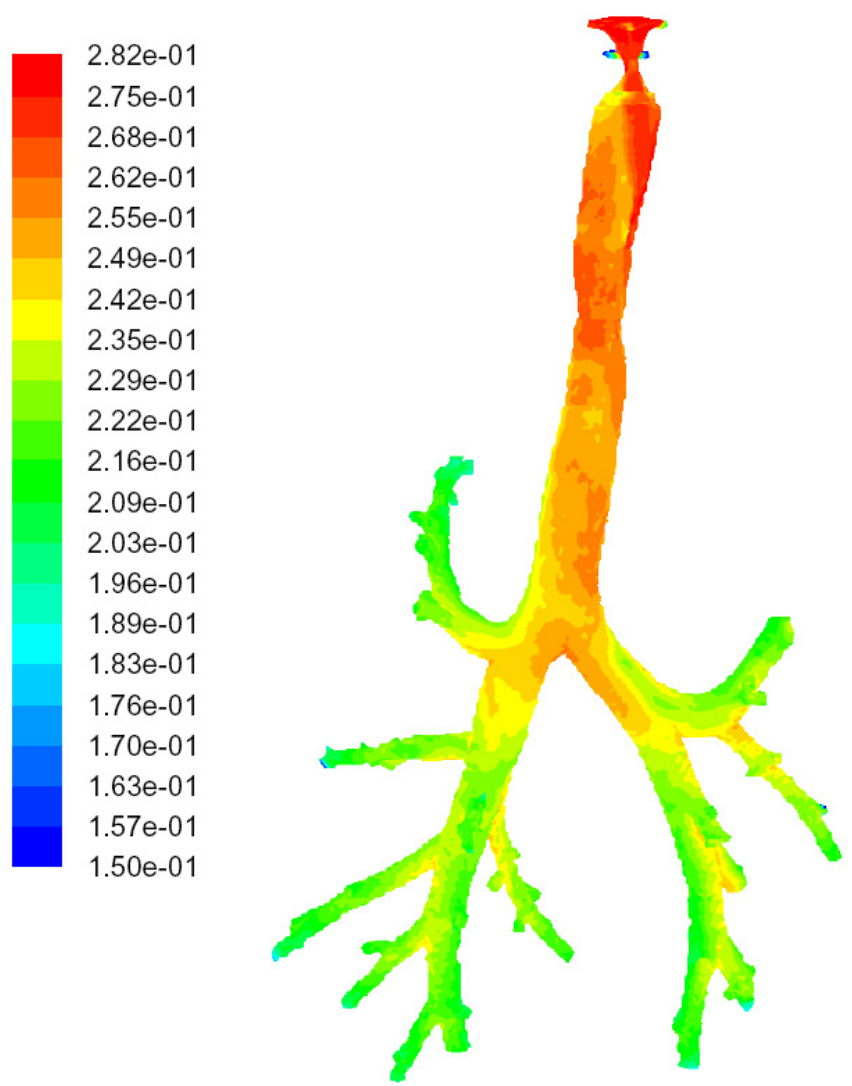

Figure 3: The $\mathrm{O}_{3}$ wall flux distribution for a slow first-order reaction with $\mathrm{Da}=$ $15(\operatorname{Re}=230, P e=195)$.

due to both the substantial uptake of ozone within the larynx, and the effect of the larynx on the downstream flow pattern.

The structure of the larynx significantly affects the resulting flow pattern in the trachea. The larynx geometry includes a narrow opening between the vocal cords (called rima glottidis) where a sharp and sudden change in the cross-sectional area of the larynx occurs. For both reaction models, the flow enters the airways with uniform velocity through the inlet of the larynx. The sudden reduction in the cross-sectional area of the larynx just upstream of the rima glottidis leads to the formation of a curved sheet-like laryngeal jet that impinges on one side of the tracheal wall a short distance downstream of the larynx (see the magnified images in Figs. 1 and 2). The bending of the laryngeal jet causes asymmetry in the flow structure extending to the distal trachea. It produces a complex flow pattern with skewed velocity profiles and recirculation regions in the trachea. It should 
be noted that the complex secondary flow patterns observed in the trachea are produced based on laminar flow simulations. Since the maximum local Reynolds number associated with the Laryngeal jet is about 1,900 for the inspiratory flow rate considered in this study, the assumption of laminar flow regime in the airways is reasonable.

The asymmetry of the three-dimensional airway structure near the first two or three bifurcations leads to significant asymmetry in partitioning of ozone among the airways downstream of those bifurcations. In addition, bending of the airways beyond those bifurcations causes the concentration profiles to be skewed toward one side of the airways. Asymmetry of the velocity and $\mathrm{O}_{3}$ concentration distributions gives rise to an asymmetric $\mathrm{O}_{3}$ wall flux distribution, as shown in Fig. 3 for the case of a slow first-order reaction. The $O_{3}$ flux distribution on the trachea wall is significantly affected by the complex flow pattern produced by the larynx. In particular, the impingement of the laryngeal jet on the tracheal wall produces a hotspot of ozone flux in the vicinity of the impingement region. Overall, the secondary flow patterns produced in the trachea by the presence of the larynx result in a more uniform $\mathrm{O}_{3}$ wall flux distribution within the trachea, compared to the corresponding simulations in the same airway structure without the larynx.

\section{Acknowledgement}

This work was supported by the National Institute of Environmental Health Sciences research grant 1 P01 ES 11617.

\section{References}

[1] Castleman, W.L., Tyler, W.S. \& Dungworth, D.L., Lesions in respiratory bronchioles and conducting airways of monkeys exposed to ambient levels of ozone. Exp Mol Pathol, 26(3), pp. 384-400, 1977.

[2] Review of national ambient air quality standards for ozone, assessment of scientific and technical information. US Environmental Protection Agency, Office of Air Quality Planning and Standards OAQPS Staff Paper EPA452/R-96-007, June 1996.

[3] Cross, C., van der Vliet, A., O’Neill, C.A., Louie, S. \& Halliwell, B., Oxidants, antioxidants, and respiratory tract lining fluids. Environ Health Perspect, 102(Suppl. 10), pp. 185-191, 1994.

[4] Chang, L., Miller, F.J., Ultman, J.S., Huang, Y., Stockstill, B.L., Grose, E., Graham, J.A., Ospital, J.J. \& Crapo., J.D., Alveolar epithelial cell injuries by subchronic exposure to low concentrations of ozone correlate with cumulative dose. Tox Appl Pharm, 109, pp. 219-234, 1991.

[5] Keshavarzi, B., Numerical Prediction of the Focal Sites of Ozone-Induced Tissue Injury in the Respiratory Tract. Ph.D. thesis, The Pennsylvania State University, 2009. 
[6] Kimbell, J.S. \& Miller, F.J., Regional respiratory-tract absorption of inhaled reactive gases: a modeling approach. In: $D$ E Gardner, J D Crapo, $R O$ Mcclellan, eds Toxicology of the Lung 3rd ed Philadelphia: Taylor \& Francis, 102, pp. 557-597, 1999.

[7] Overton, J.H., Graham, R.C. \& Miller, F.J., A model of the regional uptake of gaseous pollutants in the lung: ii. the sensitivity of ozone uptake in laboratory animal lungs to anatomical and ventilatory parameters. Tox Appl Pharm, 88(3), pp. 418-432, 1987.

[8] Miller, F.J., Menzel, D.B. \& Coffin, D.L., Similarity between man and laboratory animals in regional pulmonary deposition of ozone. Environ Res, 17(1), pp. 84-101, 1978.

[9] Miller, F.J., Overton, J.H., Jaskot, R.H. \& Menzel, D.B., A model of the regional uptake of gaseous pollutants in the lung: I. the sensitivity of the uptake of ozone in the human lung to lower respiratory tract secretions and exercise. Toxicol Appl Pharmacol, 79(1), pp. 11-27, 1985.

[10] Blackburn, K., Recommendations for and documentation of biological values for use in risk assessment. IEPA/600/6-87/008, pp. 4-16, 1996.

[11] Taylor, A.B., Borhan, A. \& Ultman, J.S., Three-dimensional simulations of reactive gas uptake in single airway bifurcation. Ann of Biomed Eng, 35(2), pp. 235-249. 